\title{
Mouthguard Controller for Unconstrained Control of External Devices
}

\author{
Koji Toma, ${ }^{1}$ Keisuke Tomoto, ${ }^{2}$ Kumi Yokota, ${ }^{1}$ Nao Yasuda, ${ }^{2}$ \\ Tatsuya Ishikawa, ${ }^{2}$ Takahiro Arakawa, ${ }^{1}$ and Kohji Mitsubayashi ${ }^{1,2^{*}}$ \\ ${ }^{1}$ Department of Biomedical Devices and Instrumentation, Institute of Biomaterials and Bioengineering, \\ Tokyo Medical and Dental University, 2-3-10 Kanda-Surugadai, Chiyoda-ku, Tokyo 101-0062, Japan \\ ${ }^{2}$ Graduate School of Medical and Dental Sciences, Tokyo Medical and Dental University, \\ 1-5-45 Yushima, Bunkyo-ku, Tokyo 113-8510, Japan
}

(Received May 31, 2018; accepted October 18, 2018)

Keywords: controller, mouthguard, tetraplegia, occlusion

A mouthguard (MG) controller with a wireless data transmitter was developed for unconstrained control of external devices. The MG controller was composed of three pairs of a pressure sensor and a Bluetooth low energy (BLE) wireless data logger for the upper and lower jaws. The pressure sensors and the BLE data loggers were sandwiched by two dental MG materials so that they were not exposed in the oral environment. Pressure applied to the sensor was measured as a resistance change, and an algorithm that exploited the sensor output to regulate the control of an external device, such as a smart device, was developed. The pressure sensor encapsulated in the MG material showed a dynamic range of 1.7-29 N. In an experiment using a jaw phantom, the response time to applied occlusal pressure was 0.18 s. Finally, the use of the measured pressure to manipulate a pointer in a smart device application was demonstrated. The results indicate that the developed MG controller enables the manipulation of devices in one's vicinity via occlusion.

\section{Introduction}

Controlling the hardware or software without spatiotemporal restriction is useful because it extends the usability of external devices that are beyond one's reach by overcoming the distance barrier. From medical and healthcare points of view, technology of unconstrained control has potential for supporting those who suffer from tetraplegia, which is also referred to as quadriplegia. Tetraplegia is paralysis in all four limbs caused by spinal cord injury or damage to the brain. Up to now, much effort has been devoted to developing technology that allows device manipulation without the use of the four limbs: for example, an eye gaze system detects corneal reflection and pupil position and uses them as input; ${ }^{(1,2)}$ a head-mounted assistive technology detects neck or shoulder movements; ${ }^{(3)}$ electromyography (EMG), a diaphragm-controlled assistive technology, also referred to as sip-and-puff, uses air pressure changes upon inhaling and exhaling through a tube as input. ${ }^{(4)}$ More recently, a brain-machine interface (BMI) and a

*Corresponding author: e-mail: m.bdi@tmd.ac.jp

https://doi.org/10.18494/SAM.2018.2014 
brain-computer interface $(\mathrm{BCI})$ have been gaining great attention. ${ }^{(5-8)}$ There are noninvasive and invasive ways to read out neuronal information, such as wearing or implanting electrodes, which allow direct communication with the brain to translate neuronal information for controlling hardware or software. ${ }^{(9-11)}$ With the BMI, wheelchairs ${ }^{(12-14)}$ and robotic arms ${ }^{(15-17)}$ have been successfully controlled. Although these technologies are promising, there are some points that should be improved, such as the complicated signal analysis, misdetection caused by head movement, spatial restriction of the camera position, and time-consuming learning process of neuronal information patterns.

Recently, we have reported on a mouthguard (MG) biosensor for monitoring saliva glucose. ${ }^{(18)}$ The MG biosensor is composed of a glucose sensor and a wireless data logger along with dental MG material, and it allows wireless and continuous measurement of glucose concentration in a buffer solution. In many cases, occlusion or tongue motion remains possible for tetraplegics, and thus, we expect that oral motion can be used to control external devices. In this study, we developed a MG controller for unconstrained manipulation of external devices by applying the basic technology of the MG biosensor. Three pairs of a pressure sensor and a Bluetooth low energy (BLE) wireless data logger were encapsulated in a dental MG material, and occlusal pressures at three different positions - front tooth and right and left second molars-were measured. After the characterization of the $\mathrm{MG}$ controller, manipulation of a pointer in a smart device application via a combination of measured occlusal pressures at three positions was demonstrated.

\section{Materials and Methods}

\subsection{Fabrication and characterization of sheet-type pressure sensor}

In the MG controller, all components, including BLE data loggers and pressure sensors, were encapsulated in the MG material of polyethylene terephthalate glycol (PETG, ERKODENT) with a thickness of $0.5 \mathrm{~mm}$. This encapsulation may alter and degrade the pressure sensor performance. Therefore, firstly, a sheet-type pressure sensor was fabricated in the same manner as the MG controller, with the exception that the sheet sensor did not include the BLE data logger, in order to investigate whether the sensor performance in the MG material is sufficient for measuring occlusal pressure. Figure 1 illustrates the fabrication process for the sheet-type pressure sensor. First, a force-sensing resistor used as a pressure sensor (FSR400, Interlink Electronics) with a sensing area diameter of $5 \mathrm{~mm}$ was laid on the first layer of the MG material. Then, the second layer of the MG material was heated and softened, followed by vacuum forming over the first layer using a vacuum-forming machine (Vacuum Adapter I, Yamahachi Dental). Finally, the pressure sensor was neatly encapsulated by heat sealing both MG materials using a heat gun (No. 880B, HAKKO).

In the characterization of the sheet-type pressure sensor, various pressures were applied to the sensing area of the pressure sensor using a compression testing machine (SV-55C, Imada Seisakusho). The sensor was placed on a stage of the machine and vertically compressed. Electric current was measured by applying a constant voltage of $400 \mathrm{mV}$ with a potentiostat (Model 1112, Husou Electrochemical System). 
(i) Applying the pressure sensor to MG material sheets

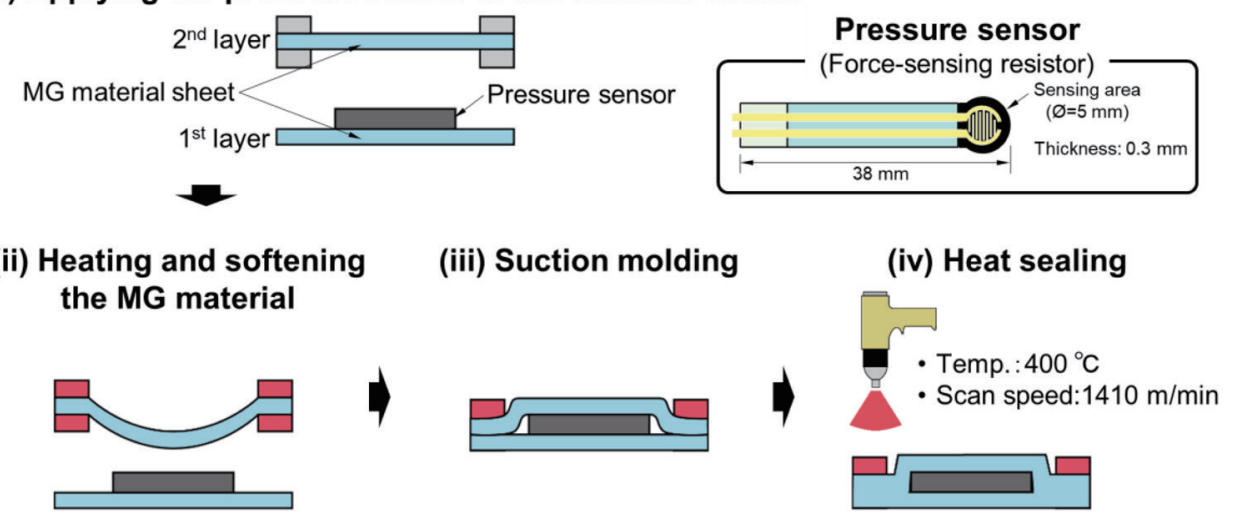

Fig. 1. (Color online) Fabrication process for sheet-type pressure sensor.

\subsection{Fabrication of MG controller}

In the fabrication of the MG controller, a tailor-made $\mathrm{MG}$ was first prepared as follows: alginate impression material (Aroma Fine Plus, GC) was mixed well with water until the material was homogeneously viscous; an impression tray filled with alginate impression material was inserted and allowed to set in the mouth for 3 min to produce a dental impression; dental stone plaster (Zo-Stone, Shimomura Gypsum) was well mixed with water and poured into the dental impression; after $1 \mathrm{~h}$, the plaster was removed from the dental impression and trimmed; PETG MG material was vacuum formed, as illustrated in Fig. 1, over the plaster. After making a MG, pressure sensors and BLE data loggers were implemented. For the lower jaw, two pairs of a pressure sensor and a custom-made BLE data logger (Wavetech) were placed at the positions of the left and right second molars on the fabricated MG, and then covered with the second layer of MG material by vacuum forming so that neither the pressure sensor nor the BLE data logger was exposed in the mouth [Fig. 2(a)]. For the upper jaw, similarly to the lower jaw, one pair of the pressure sensor and the BLE data logger was implemented at the position of a front tooth [Fig. 2(b)].

\section{Results and Discussion}

\subsection{Sensitivity of sheet-type pressure sensor}

Figure 3 shows a calibration curve of a sheet-type pressure sensor. In the pressure range from 1.7 to $50.2 \mathrm{~N}$, the plots can be fitted by the following equation with a correlation coefficient $R=0.996$ :

$$
\text { output }(\mu \mathrm{A})=2.4[\text { force }] /(1.38+[\text { force }])
$$

where [force] is the force applied to the sheet-type pressure sensor in N. The determined 


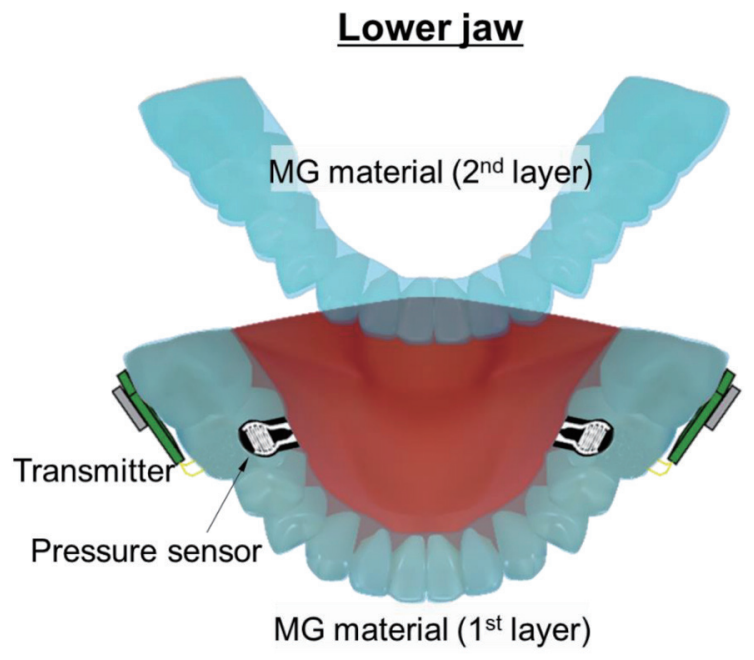

(a)

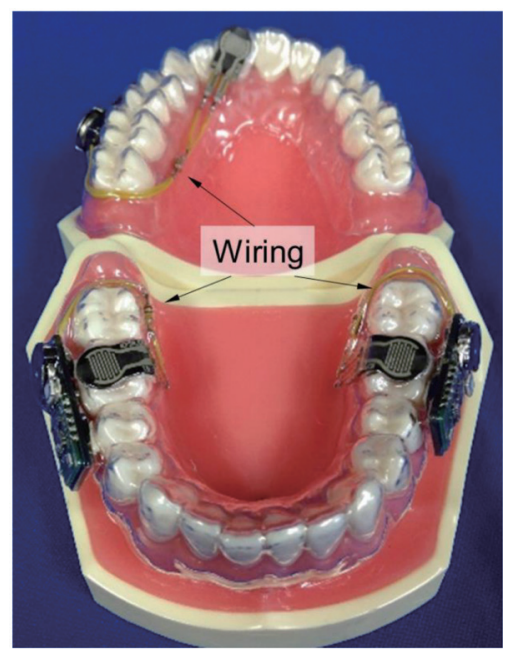

(b)

Fig. 2. (Color online) (a) Schematic image of MG controller components for lower jaw. (b) Fabricated MG controller with a jaw phantom. One pair of a pressure sensor and a BLE data logger are implemented at each of the positions of a front tooth on the upper jaw and right and left second molars on the lower jaw.

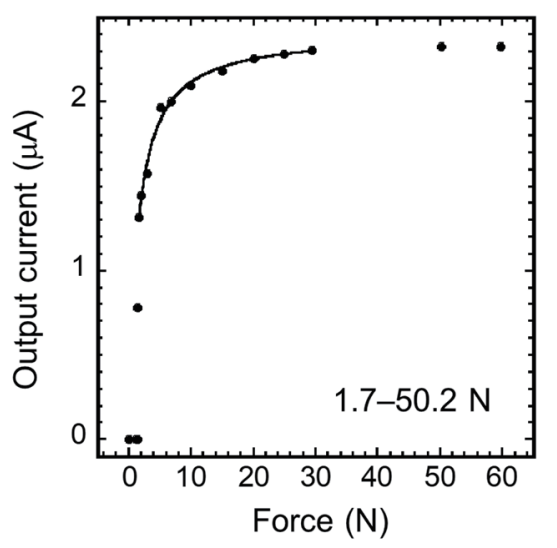

Fig. 3. (Color online) Calibration curve of the sheet-type pressure sensor. Force was applied to the sensor using the compression testing machine.

dynamic range of the sheet-type pressure sensor, which was $1.7-50.2 \mathrm{~N}$, indicates that the pressure sensor encapsulated by MG material can measure weak occlusion force because the typical force induced by mastication is $200 \mathrm{~N}^{(19)}$

\subsection{Characteristics of pressure sensors in MG controller}

The fabricated MG controller was characterized using a jaw phantom [Fig. 4(a)]. Force was applied to the MG controller, which was attached to the phantom, by opening and closing the phantom. Figures 4(b) and 4(c) show the time courses of output current generated by constant 

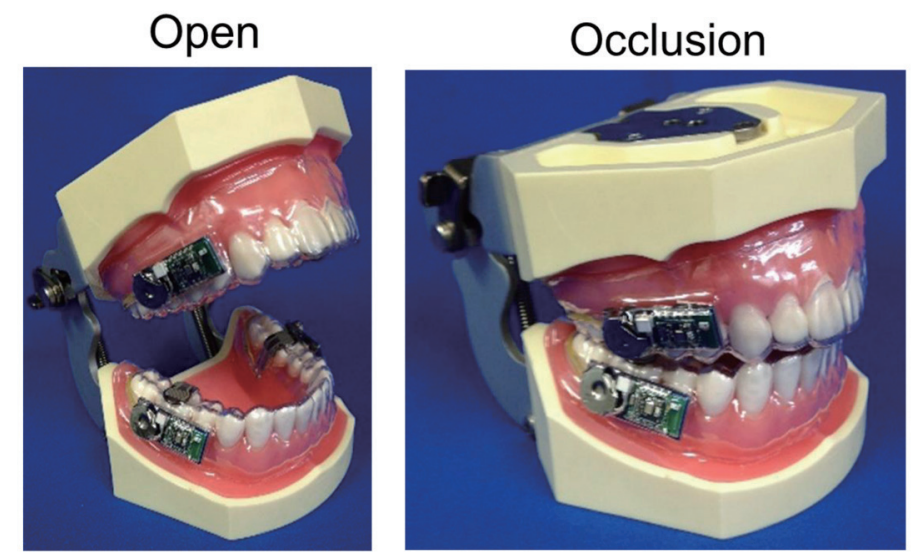

(a)

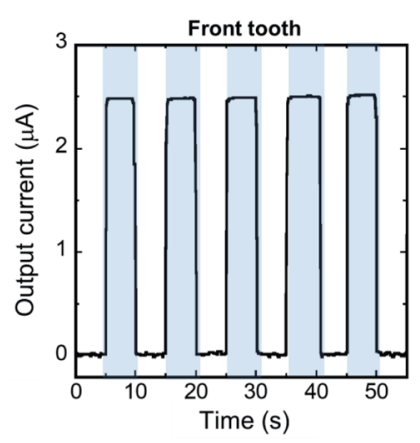

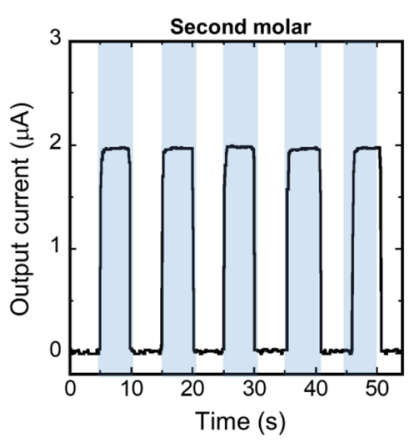

(b)
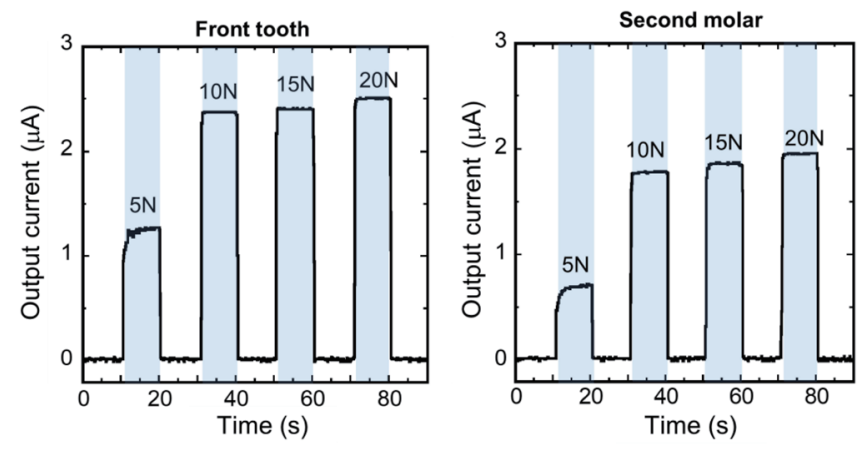

(c)

Fig. 4. (Color online) (a) Open and occluded jaw phantom. (b) Repeated measurements of output current at constant and (c) varied occlusal pressures.

or varied force. When a constant force of $20 \mathrm{~N}$ was applied [Fig. 4(b)], the output current was higher for the front tooth than that for the second molar. The $90 \%$ response time was also shorter for the front tooth $(0.18 \mathrm{~s})$ than that for the second molar $(0.33 \mathrm{~s})$. Both output currents were highly reproducible, as indicated by the coefficients of variation (C.Vs.) of $0.48 \%$ for the front tooth and $0.36 \%$ for the second molar for five repeated measurements. When various forces from 5 to $20 \mathrm{~N}$ were applied [Fig. 4(c)], the second molar showed a higher output current than the front tooth, although both parts were capable of differentiating between weak and strong occlusions. This output difference may be attributed to the inhomogeneous pressure distribution caused by the arrangement of the teeth and different structure of each tooth. Therefore, a calibration curve for each sensor position is required to improve the accuracy of measurement.

\subsection{Manipulation of pointer in smart device application}

Before manipulating an external device via the MG controller, it was examined whether two pairs of a pressure sensor and a BLE data logger can measure pressure and transmit the 
measured data to an external device without any trouble. Here, a smart device (Android tablet) was used to receive transmitted data. Figure 5(a) shows a schematic image of data communication between three pairs of a BLE data logger and a smart device occuring in the MG controller. Data from a BLE data logger were transmitted with a time interval of $30 \mathrm{~s}$, and data communication from each BLE data logger took place. Figure 5(b) shows the output current wirelessly transmitted to the Android smart device when a given pressure was applied to two pairs of pressure sensors. It was demonstrated that even when two BLE data loggers communicated with the smart device simultaneously, measured data were transmitted successfully. Hence, it was confirmed that simultaneous measurement and wireless data communication by multiple pressure sensors and BLE data loggers are possible.

Now the MG controller can be applied to move a pointer in the smart device. Table 1 shows the algorithm used for pointer manipulation. When the measured current exceeds the threshold of $2.0 \mu \mathrm{A}, 1$ is returned. Likewise, when the measured current is smaller than the threshold, the result is 0 . Finally, a combination of three results - front tooth and left and right second molars - determines the direction of motion of the pointer.

Images of pointer manipulation via the MG controller are shown in Fig. 6. The measured pressure on the MG controller was wirelessly transmitted to the smart device, and the pointer in an application of the smart device was moved on the basis of the algorithm described in
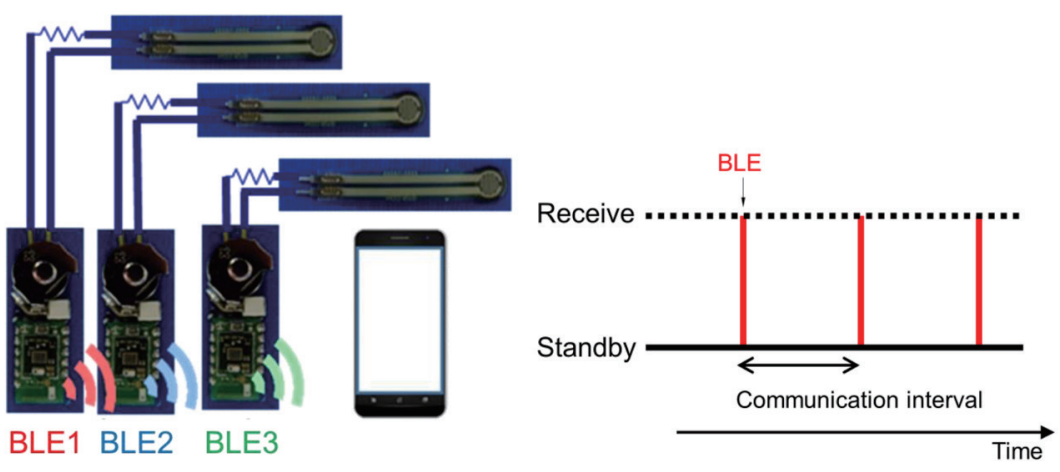

(a)

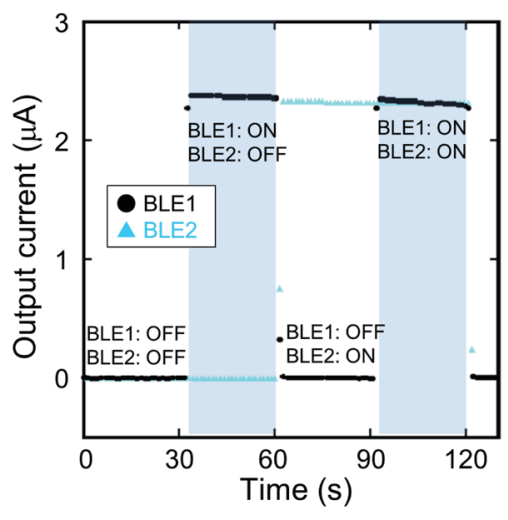

(b)

Fig. 5. (Color online) (a) Schematic illustration of simultaneous measurement and wireless data communication with a smart device. (b) Pressure measurement and data transmission by two pairs of a pressure sensor and a BLE data logger.

Table 1

Algorithm determining the direction of motion of a pointer.

\begin{tabular}{lccc}
\hline Front tooth & Left second molar & Right second molar & Motion \\
\hline 0 & 1 & 0 & $\leftarrow$ \\
0 & 0 & 1 & $\rightarrow$ \\
0 & 1 & 1 & $\downarrow$ \\
1 & 0 & 0 & $\uparrow$ \\
\hline
\end{tabular}




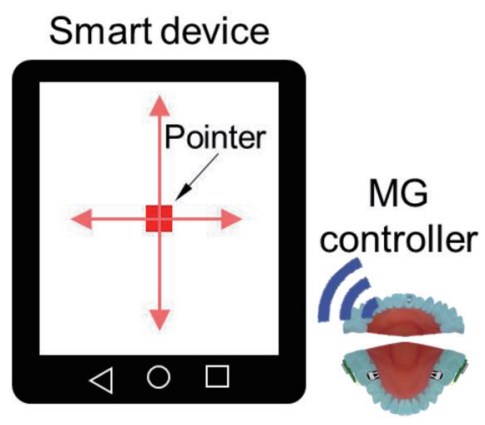

(a)

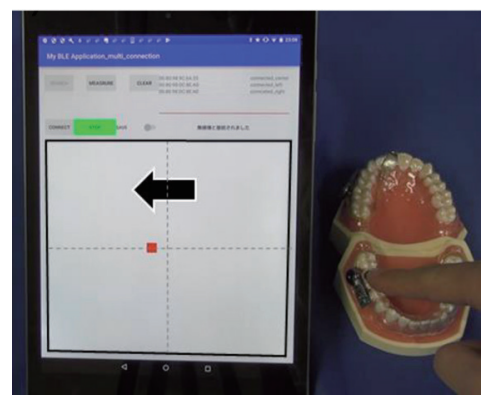

(c)

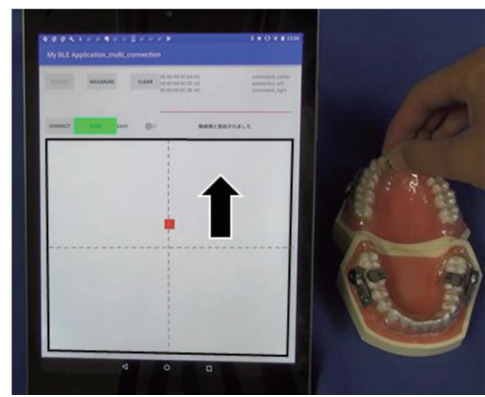

(e)

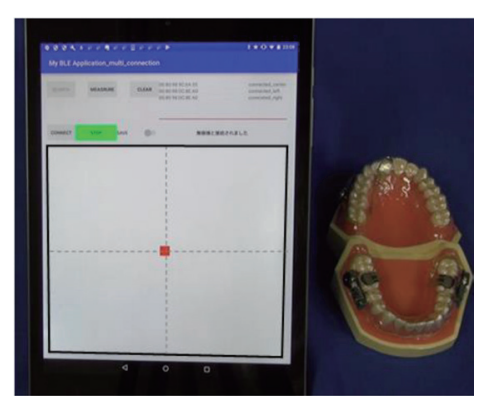

(b)

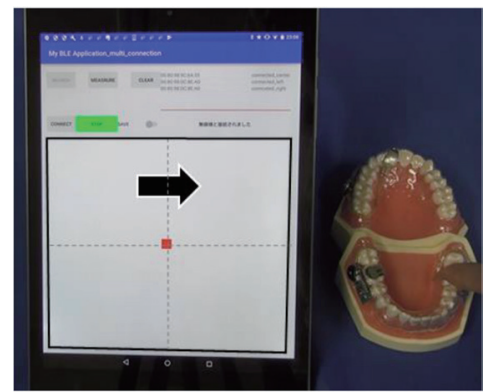

(d)

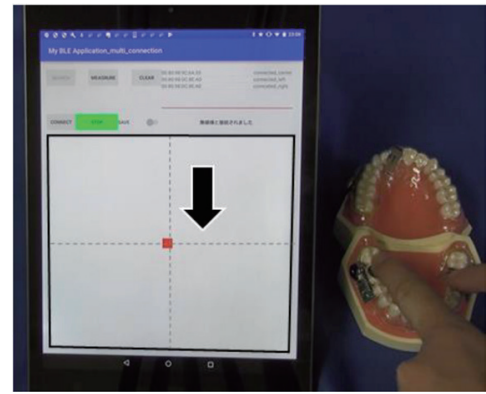

(f)

Fig. 6. (Color online) (a) Schematic illustration of the experimental setup for demonstration of pointer manipulation in a smart device using a MG controller. (b) Initial position of the pointer. Various pointer motions corresponding to the pressures: (c) moving left with pressure on left second molar; (d) moving right with pressure on right second molar; (e) moving up with pressure on front tooth; (f) moving down with pressure on both second molars.

Table 1: the pointer moved left from the original center position when force was applied to the pressure sensor on the left second molar [Figs. 6(b) and 6(c)]. Similarly, other pointer motions were also demonstrated by applying force to corresponding pressure sensors [Figs. 6(d)-6(f)]. These results show the high utility of the MG controller and the possibility of more complex manipulation of external devices. 


\section{Conclusions}

We reported a MG controller for unconstrained manipulation of external devices. Three pairs of a pressure sensor and BLE data logger were encapsulated in dental MG material. Using a jaw phantom, the measurement of occlusion forces and wireless data communication with a smart device were demonstrated. Moving a pointer in the smart device on the basis of an algorithm that utilizes a combination of occlusion forces at different tooth positions indicated the great potential of the MG controller for use in device manipulation. In future work, the performance of the MG controller will be further improved, and it will be applied to technologies for assisting tetraplegic patients.

\section{Acknowledgments}

This work was supported by Japan Radio Co., Ltd., the Japan Society for the Promotion of Science (JSPS) Grant-in-Aid for Scientific Research System, the Japan Science and Technology Agency (JST), and the Ministry of Education, Culture, Sports, Science and Technology (MEXT) Special Funds for "Cooperative Research Project of Research Center for Biomedical Engineering".

\section{References}

1 Z. Ramdane-Cherif and A. Nait-Ali: IEEE Trans. Instrum. Meas. 57 (2008) 716.

2 T. E. Hutchinson, K. P. White, W. N. Martin, K. C. Reichert, and L. A. Frey: IEEE Trans. Syst. Man. Cybern. 19 (1989) 1527.

3 M. R. Williams and R. F. Kirsch: IEEE Trans. Neural Syst. Rehabil. Eng. 16 (2008) 485.

4 M. Jones, K. Grogg, J. Anschutz, and R. Fierman: Assist. Technol. 20 (2008) 107.

5 P. Nair: Proc. Natl. Acad. Sci. U.S.A. 110 (2013) 18343.

6 M. A. Lebedev and M. A. L. Nicolelis: Trends Neurosci. 29 (2006) 536.

7 F. Cincotti, D. Mattia, F. Aloise, S. Bufalari, G. Schalk, G. Oriolo, A. Cherubini, M. G. Marciani, and F. Babiloni: Brain Res. Bull. 75 (2008) 796.

8 F. Galán, M. Nuttin, E. Lew, P. W. Ferrez, G. Vanacker, J. Philips, and J. del R. Millán: Clin. Neurophysiol. 119 (2008) 2159.

9 P. R. Kennedy and R. A. Bakay: Neuroreport 9 (1998) 1707.

10 E. C. Leuthardt, G. Schalk, J. R. Wolpaw, J. G. Ojemann, and D. W. Moran: J. Neural Eng. 1 (2004) 63.

11 J. P. Donoghue: Nat. Neurosci. 5 (2002) 1085.

12 E. B. Thorp, F. Abdollahi, D. Chen, A. Farshchiansadegh, M.-H. Lee, J. P. Pedersen, C. Pierella, E. J. Roth, I. Seanez Gonzalez, and F. A. Mussa-Ivaldi: IEEE Trans. Neural Syst. Rehabil. Eng. 24 (2016) 249.

13 J. D. R. Millan, F. Galan, D. Vanhooydonck, E. Lew, J. Philips, and M. Nuttin: 2009 Annu. Int. Conf. IEEE Engineering in Medicine and Biology Society (2009) 3361.

14 G. N. Jayabhavani, N. R. Raajan, and R. Rubini: 2013 IEEE Conf. Information and Communication Technologies (2013) 1129.

15 E. Hortal, D. Planelles, A. Costa, E. Iáñez, A. Úbeda, J. M. Azorín, and E. Fernández: Neurocomputing 151 (2015) 116.

16 G. Onose, C. Grozea, A. Anghelescu, C. Daia, C. J. Sinescu, A. V. Ciurea, T. Spircu, A. Mirea, I. Andone, A. Spânu, C. Popescu, A.-S. Mihăescu, S. Fazli, M. Danóczy, and F. Popescu: Spinal Cord 50 (2012) 599.

17 Y. J. Kim, S. W. Park, H. G. Yeom, M. S. Bang, J. S. Kim, C. K. Chung, and S. Kim: Biomed. Eng. Online 14 (2015) 81.

18 T. Arakawa, Y. Kuroki, H. Nitta, P. Chouhan, K. Toma, S. Sawada, S. Takeuchi, T. Sekita, K. Akiyoshi, S. Minakuchi, and K. Mitsubayashi: Biosens. Bioelectron. 84 (2016) 106.

19 G. Ortuğ: Ann. Anat. 184 (2002) 393. 